\title{
THE BULGE OF THE MILKY WAY AND COSMIC RAYS
}

\author{
F.Jansen ${ }^{1}$, K.-P.Wenzel ${ }^{1}$, D.O'Sullivan ${ }^{2}$, A. Thompson ${ }^{2}$ \\ 1.Space Sci. Dept. of ESA, ESTEC, Noordwijk, NL \\ 2.Dublin Inst.for Adv.Studies, Dublin, Ireland
}

\begin{abstract}
The propagation of cosmic ray protons and antiprotons from the inner Galaxy via the galactic halo to the Sun supplies a good agreement with the observed cosmic ray gradient and is in the order of the measured anti-proton flux. Ultra heavy cosmic ray nuclei may have the same origin.
\end{abstract}

\section{Introduction}

The near-infrared image of the Milky Way obtained by DIRBE on the satellite COBE lead to the question of the contribution from cosmic ray sources within the "peanut-shaped" bulge to the cosmic ray flux at the sun. The trend of the preliminary results from the DUBLIN-ESTEC experiment on LDEF satellite (O'Sullivan et al., 1992) is a confirmation of earlier measurements from Ariel 6 and HEAO-3. Therefore cosmic ray nuclei with charge greater than 60 disagree with the predictions of the standard source and propagation models of cosmic rays. For instance in the leaky Box Model such ultra heavy cosmic ray nuclei are a factor of two less abundant than observed (Fowler et al. 1987). The observed anti-protons are a factor of three over abundant than predicted in the LBM ( $\mathrm{Tan}$ and $\mathrm{Ng}, 1983$ ). In both cases the mean thickness traversed by cosmic rays before reaching the sun position in the Galaxy was taken to be $7 \mathrm{gcm}^{-2}$ as measured for ${ }^{10} \mathrm{Be}$ cosmic ray nuclei. Moreover the gamma-ray bulge detected by Cos-B mission indicated the existence of a cosmic ray gradient between the inner region of the Galaxy and the sun of about 1,5 (Strong et al., 1988). This cosmic ray gradient disagrees with one of the basic assumptions in the LBM that there is a homogeneous distribution of cosmic rays in the Galaxy. A significant mixing of cosmic rays in the galactic halo must occur, because the observations at $408 \mathrm{MHz}$ show in the inner Galaxy many spurs out of the galactic plane (Sofue, 1988).

\section{The halo diffusion model and the results}

The aim of the proposed Halo Diffusion Model for cosmic ray protons and antiprotons by Halm et al. (1992a,b) was to build a model under conditions as close as possible to those described in the introduction. A diffusion equation for the cosmic ray density $\mathrm{N}$ was solved

$$
-\frac{D(E)}{r} \frac{\partial^{2}}{\partial r^{2}}(r N(r, E))+\frac{\partial}{\partial E}(I(E) N(r, E))+\Sigma(E) N(r, E)=q(E) e^{-\left(r / R_{0}\right)^{2}}
$$


(From left to right the terms for diffusion, ionization, annihilation and the source term, $E$ is the total energy, the radial coordinate $r$ is directed along the path of a cosmic ray particle with $R_{0}$ as the cosmic ray source parameter.). The calculated and the observed cosmic ray gradient are in agreement if $r / R_{0} \approx 1,05(r=10 \mathrm{kpc}$ for the position of the sun.). The gradient is less dependent on the diffusion constant $D_{0}$ in $\mathrm{D}(\mathrm{E})$. With $\mathrm{D}_{0} \leq 510^{28} \mathrm{~cm}^{2} \mathrm{~s}^{-1}$ (Bykov and Toptygin, 1990) and from the equation

$$
n=2 D_{0} x_{0} /\left(R_{0} m v\right)
$$

the gas particle density $n$ traversed by relativistic cosmic rays is smaller than $10^{-2} \mathrm{~cm}^{-3}$ (m proton rest mass, $\mathrm{v}$ velocity of the cosmic rays). This value agrees with the observation mentioned above, that the cosmic ray quickly leave the region of the inner Galaxy and propagate mainly via the halo to the sun. With $D_{0}=510^{28} \mathrm{~cm}^{2} \mathrm{~s}^{-1}$ the flux of anti-protons, which are produced during the propagation by collisions of cosmic ray protons with the interstellar gas is in the order of the LBM. In contrast to the protons the anti-proton flux strongly depends on $D_{0}$. For instance a value of $510^{27} \mathrm{~cm}^{2} \mathrm{~s}^{-1}$ gives about three times more antiprotons.

Because it was shown by Halm et al. (1992a) for antiprotons that the LBM and the HDM solutions are similar for $r / R_{0} \approx 1$ and because of the increasing anti-proton flux with decreasing diffusion constant it may be possible to produce an ultraheavy cosmic ray abundance in the HDM which is similar to, or greater than, that in the LBM.

\section{References}

Bykov, A.M.; Toptygin, I.N.: 1990, Proc. 21th Intern. Conf. Cosmic Rays 3, 307

Fowler, P.H.; Walker, R.N.F.; Masheder, M.R.W.; Moses, R.T.; Worley, A.; Gay, A.M.: 1987, Astrophys. J. 314, 739

Halm, I.; Jansen, F.; de Niem, D.: 1992a, Astron. Astrophys., in press

Halm, I.; Jansen, F.; de Niem, D.: 1992b, Astron. Astrophys., subm.

O 'Sullivan, D.; Thompson, A.; Bosch, J.; Keegan, R.; Wenzel, K.-P.; Jansen, F.; Domingo, C.: 1992, COSPAR Washington, in prep.

Sofue, Y.: 1988, Publ. Astron. Soc. Japan 40, 567

Strong, A.W.; Bloemen, J.B.G.M., Dame, T.M., Grenier, I.A. ; Hermsen, W.; Lebrun, F.; Nyman, L.-A.; Pollock, A.M.T.; Thaddeus, P.:1988, Astron. Astrophys. 207, 1

Tan,L.C.; Ng, L.C.: 1983, J. Phys. G: Nucl. Phys. 9, 227 